\title{
Efetividade do aparelho ortopédico Bionator de Balters no tratamento do ronco e apnéia do sono
}

\author{
Paulo Alberto Donadelli Nabarro*, Roberta Tarkany Basting Höfling**
}

\begin{abstract}
Resumo
Objetivos: avaliar a efetividade do aparelho ortopédico Bionator de Balters no tratamento da Síndrome da Apnéia Obstrutiva do Sono (SAOS). Metodologia: foram selecionados 16 pacientes (18,8\% do gênero feminino e $81,3 \%$ do masculino) que apresentaram a SAOS mediante diagnóstico. Dos 16 pacientes que utilizaram o aparelho ortopédico Bionator de Balters, apenas 9 apresentaram boa aceitação $(56,25 \%)$ e concluíram o tratamento. Estes foram submetidos a nova polissonografia, realizada com o paciente fazendo uso do aparelho ortopédico Bionator de Balters, para avaliar a eficácia deste dispositivo no tratamento do ronco e da apnéia. A comparação entre os resultados do IDR (índice de distúrbio respiratório) inicial $(11,5)$ e final $(4,2)$ foi realizada utilizando-se o teste t pareado $(\alpha=0,05)$. Resultados: observou-se melhora estatisticamente significativa do IDR após a utilização do dispositivo. Conclusões: a utilização do aparelho ortopédico Bionator de Balters pode ser considerada um método de tratamento da SAOS em casos leves ou moderados, apesar de ser um método pouco tolerado pelos pacientes.
\end{abstract}

Palavras-chave: Bionator de Balters. Apnéia. Ronco.

\section{INTRODUÇÃO}

Dormimos um terço do tempo de nossas vidas e, durante o sono, é difícil perceber que processos patológicos - que não causam suficiente desconforto para nos manterem acordados - estão ocorrendo com o nosso corpo $^{31}$. Como não estamos conscientes, não percebemos que estamos doentes, principalmente nos casos da Síndrome da Apnéia Obstrutiva do Sono (SAOS).

A SAOS é uma doença crônica, progressiva, incapacitante e com conseqüências ameaçadoras sobre o potencial de vida. Caracteriza-se por um colapso das vias aéreas superiores, devido ao posicionamento posterior da língua, diminuindo o fluxo aéreo ${ }^{33}$. A SAOS apresenta como sinais e sintomas o ronco, a interrupção da respiração de forma intermitente durante o sono, agitação ao dormir, sensação de sufocamento ao despertar, sonolência diurna excessiva, impotência sexual, dores de cabeça e irritabilidade. Na maioria dos casos, o indivíduo percebe algum tipo de comprometimento de sua saúde quando acorda com o ruído do ronco após a respiração ter parado segundos antes ${ }^{11,18}$.

O ronco é um ruído produzido pela vibração do

* Especialista em Ortopedia Funcional dos Maxilares e Mestre em Ortodontia pelo Centro de Estudos Odontológicos São Leopoldo Mandic-

Campinas. Pós-graduando em Medicina do Sono pela UNIFESP/Instituto do Sono/AFIP - São Paulo.

** Mestre e doutora em Clínica Odontológica Integrada - Dentística pela Faculdade de Odontologia de Piracicaba - UNICAMP. 
palato mole e/ou de outros tecidos bucofaríngeos, durante a passagem de ar, na função respiratória. A diminuição do calibre das vias aéreas superiores, durante a inspiração normal, impõe aumento da velocidade do ar, o que favorece a vibração dos tecidos moles da faringe, levando ao ronco. É mais freqüente entre os homens do que entre as mulheres e agrava-se com o passar da idade i,19,20,21. $^{\text {. }}$

Alguns tratamentos podem ser indicados para a SAOS, incluindo a realização de técnicas cirúrgicas (uvulopalatofaringoplastia, avanços maxilomandibulares, trações do genioglosso, tireoidexia e traqueostomia, entre outras), bem como a realização de procedimentos mais conservadores, como a indicação de aparelhos funcionais que promovam o aumento da dimensão vertical de oclusão ou anteriorizacão mandibular (Bionator de Balters ou ativador elástico de Klammt) e reabilitações protéticas e/ou restauradoras ${ }^{28}$. Neste aspecto, o tratamento através de dispositivos intrabucais tem se mostrado interessante, devido ao baixo custo, facilidade de confecção e conforto ao paciente ${ }^{8}$, despertando a importância do cirurgião-dentista como um recurso humano necessário para o tratamento multidisciplinar desta doença ${ }^{5}$.

\section{REVISÃO DE LITERATURA}

A SAOS é uma patologia que afeta as vias aéreas superiores e a função cardiorespiratória do indivíduo. É definida como a repetida cessação da respiração durante o sono, com persistência do esforço respiratório, caracterizada pela obstrução ou colapso ao nível das vias aéreas superiores. Relatada como uma desordem do sono, esta patologia não se manifesta durante a vigília. Podem ser citadas como causas prováveis da apnéia obstrutiva noturna a obesidade, o estreitamento estrutural das vias aéreas superiores - como hipertrofia adenotonsilar, excesso de tecido faríngeo, micrognatia, macroglossia, obstrução nasal (desvios de septo, hipertrofia de cornetos, pólipos nasais), neoplasia faríngea, bócio e alergia -, a distrofia muscular (principalmente do gradil costal), a ingestão de álcool, a administração de benzodiazepínicos ou testosterona e o hipotireoidismo. O tamanho das vias aéreas é, também, afetado pela morfologia craniofacial, ocorrendo no estreitamento das vias aéreas e concomitante apnéia noturna em pacientes com significante retrognatia. Um padrão esqueletal anormal, que reduz as dimensões das vias aéreas durante o repouso (vigília), pode predispor a uma obstrução das vias aéreas superiores durante o sono, já que existe uma íntima relação da musculatura faríngea em relação à estrutura óssea.

A apnéia obstrutiva tem diferentes níveis de gravidade, podendo apresentar-se desde uma forma discreta, progredindo em escala crescente até níveis severos, que colocam em risco a vida do paciente. Desta forma, caracteriza uma patologia que envolve a morbidade e a mortalidade. As queixas mais comuns de pacientes que sofrem de apnéia obstrutiva noturna são a hiperssonolência diurna ( $80 \%$ dos pacientes) e o sono agitado, caracterizado por ronco forte e despertares freqüentes ${ }^{11}$.

\section{Diagnóstico do ronco noturno e da síndrome da apnéia obstrutiva do sono}

A monitorização polissonográfica procura verificar características normais ou patológicas do sono, procurando-se manter o sono em condições o mais próximo possivel das normais, semelhantes às do paciente em sua casa. Para isso, o ambiente onde será realizada a monitorização deve ser agradável (Fig. 1), com cama, temperatura, luminosidade e atenuação de sons adequados ${ }^{16}$.

\section{Tratamento}

Antes de se iniciar um tipo de tratamento, deve-se analisar a existência de obstruções na bucofaringe, especialmente na região retropalatina. Estas podem envolver uma ou mais áreas da faringe, sendo que as obstruções podem mudar de posição durante o sono, interferindo diretamente com o tipo de tratamento a ser adotado ${ }^{2}$. Enquanto Morrison et al. ${ }^{22}$ observaram obstruções limitadas à área retropalatina em 18\% dos pacientes, 


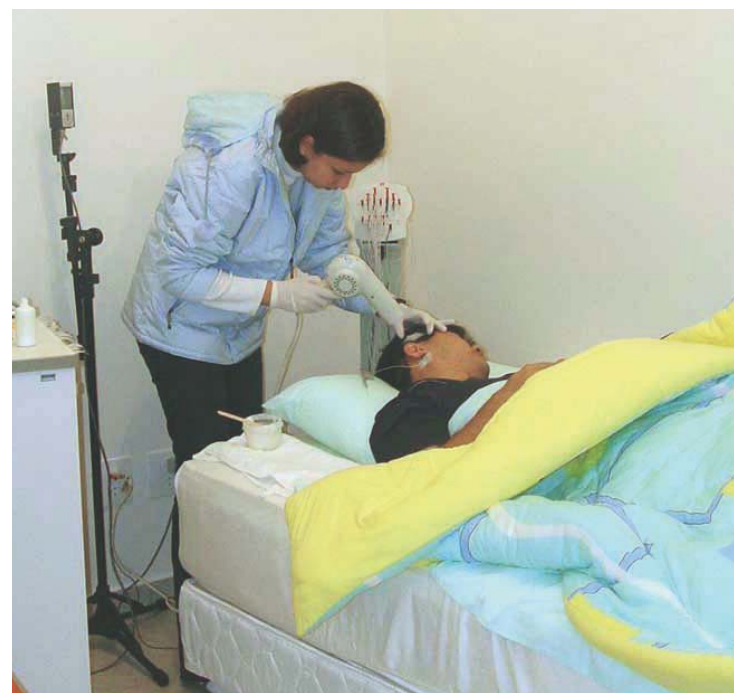

FIGURA 1 - Ambiente agradável para o exame de polissonografia, realizado por meio de aparelho polissonográfico computadorizado.

Shepard e Thawley ${ }^{29}$ mostraram que tais obstruções ocorrem na região retropalatina em $56 \%$ dos casos, durante a fase NREM do sono, e na região retrolingual em 44\%. Porém, durante o sono REM, as obstruções predominam na região retrolingual, incidindo em $78 \%$ dos pacientes.

Em relação às estratégias de tratamento, Paulin et al..$^{23}$, Ramos e Furquim ${ }^{24}$ relatam que devem ser baseadas no alívio dos sintomas, na redução da morbidade e na diminuição da mortalidade. Entretanto, deve-se melhorar a qualidade de vida do paciente $^{14}$.

Vários procedimentos terapêuticos podem ser realizados para o tratamento da SAOS e a redução do ruído do ronco, sendo classificados como não-conservadores (cirúrgicos) e conservadores ${ }^{31}$. Dentre os conservadores, podem ser citados os aparelhos intrabucais - recomendados para pacientes diagnosticados num estágio precoce (quando se pode prevenir o desenvolvimento de complicações relacionadas à apnéia obstrutiva noturna), para formas de apnéia suaves e moderadas, para formas resistentes ao tratamento cirúrgico e para pacientes que não aceitam ou não podem custear uma cirurgia ${ }^{11}$.
Os aparelhos devem ser usados durante a noite, para modificar o "corredor" de passagem do ar nas vias aéreas superiores - a fim de eliminar as obstruções. O principal mecanismo de ação dos aparelhos intrabucais é o de avanço do músculo genioglosso e aumento das vias aéreas superiores. Esses dados são constatados nos estudos com cefalometria, tomografia e ressonância magnética. Aumentar o calibre das vias aéreas superiores é possível, porque os músculos da língua se inserem na mandíbula. Assim, com o avanço da mandíbula, a base da língua afasta-se da parede posterior da faringe, permitindo, ainda, o deslocamento anterior do osso hióide e algum avanço do palato mole. Recupera-se, assim, o calibre das vias aéreas e reduz-se o colapso das mesmas e a resistência à passagem de ar. $\mathrm{O}$ aparelho deve ter boa adaptação e retenção em ambos os arcos. Caso contrário, com o relaxamento muscular do próprio sono, o paciente pode abrir a boca e a mandíbula voltar à posição original. $\mathrm{O}$ aparelho deve, ainda, ser facilmente ajustado, para que a mandíbula avance gradativamente: a mudança de postura mandibular deve ser a menor possível, porém capaz de produzir os efeitos desejados. Busca-se, assim, a proteção dos dentes, de suas estruturas de suporte e das articulações temporomandibulares ${ }^{30}$.

Desta forma, para o tratamento odontológico na SAOS, estão indicados para aumento da dimensão vertical: placas de levante, aparelhos funcionais protéticos e cosméticos; aparelhos para avanço mandibular (Bionator de Balters e ativador elástico de Klammt, dentre outros); e reabilitações (próteses totais, próteses parciais, Dentística Restauradora e implantes) ${ }^{28}$.

Sergi e Zentner ${ }^{27}$ realizaram um estudo para avaliar a influência da forma e do modelo de diferentes tipos de aparelhos funcionais, avaliando a aprovação dos pacientes. Para cada 10 voluntários envolvidos na pesquisa, oito aparelhos funcionais diferentes foram fabricados, apresentando uma variação na base da resina e na quantidade 
de abertura interoclusal. Os efeitos dos aparelhos na fala e a aceitação no início e após o uso foram avaliados por meio de testes padronizados. Entre os aparelhos testados, o Bionator, o regulador funcional FR-1 e o ativador aberto elástico demonstraram a maior aceitação dos indivíduos avaliados.

\section{MATERIAL E MÉTODOS Seleção da amostra}

Dezesseis pacientes com queixa de roncopatia e sonolência diurna excessiva foram selecionados para o experimento, após procurarem o médico otorrinolaringologista. Estes apresentavam idade entre 35 e 61 anos (média de 45,12 anos), sendo três $(18,8 \%)$ do gênero feminino e $13(81,3 \%)$ do gênero masculino. A propedêutica médica incluiu endoscopia nasofaríngea rígida e flexível, com realização de manobra de Muller em posição sentada e supina, e constatação diagnóstica de língua retro posicionada - que obliterava a passagem de ar, ocasionando o ruído denominado ronco.

A suspeita diagnóstica de apnéia foi confirmada através da monitorização polissonográfica, que verifica características normais ou patológicas do sono, procurando manter o sono em condições o mais próximo possivel das normas, semelhantes às do paciente em sua casa. Durante a polissonografia, foram mensurados os movimentos torácicos ou abdominais, o volume de ar corrente e a saturação sanguínea de oxigênio por meio de aparelho polissonográfico computadorizado (Polígrafo Ati Nautilus) (Fig. 1).

Com esses dados, foram determinados o índice de apnéia e de hipopnéia, o índice de apnéia + hipopnéia (IDR) e a saturação basal média e mínima de oxigênio.

Para a indicação do tratamento com uso de dispositivos intrabucais, o IDR deveria se situar entre 5 e 15 ou entre 15 e 30, considerados graus leve ou moderado de ronco e apnéia do sono. Portanto, o médico otorrinolaringologista determinava que o paciente apresentou SAOS, encaminhando-o para a participação neste estudo.

Durante o exame anamnésico e clínico odontológico, incluíam-se indagações sobre a ocorrência de ronco pesado, períodos de parada respiratória relatados pelo companheiro, asfixia, sonolência diurna excessiva, sono fragmentado por despertares freqüentes, sono não-reparador, chutes e movimentos de pernas durante o sono, dor de cabeça matinal, mudanças de personalidade, desempenho pobre no trabalho, perda de agilidade e memória nebulosa. Questionou-se sobre deterioração intelectual, acidentes ocupacionais, impotência sexual ou diminuição da libido, bruxismo, uso de sedativos para conciliar o sono, uso regular de anti-histamínicos, peso excessivo, idade, irregularidades cardíacas e pressão sanguínea alta.

Após obtenção de completa história médica e dentária, foram examinados os tecidos moles, incluindo a língua e a garganta. Observou-se o periodonto, as relações ortopédicas, a ATM, a oclusão, a presença de hábitos bucais, os dentes e as restaurações. Ainda foi avaliada a presença de hipertrofia das tonsilas, tamanho e posição da língua, palato mole e úvula e as relações entre essas estruturas.

\section{Confecção do dispositivo (Bionator)}

Baseado no trabalho de Sergi e Zentner ${ }^{27}$, cujo objetivo foi avaliar a influência da forma e do modelo de diferentes tipos de aparelhos funcionais, e cujos resultados mostraram que o Bionator foi o dispositivo melhor aceito pelos pacientes; o mesmo foi eleito para a atual pesquisa, dentre os aparelhos intrabucais para o tratamento da SAOS.

Para a confecção do aparelho, foram moldados ambos os arcos dentários do paciente, com hidrocolóide irreversível alginato para impressão (Jeltrate, Dentsply Indústria e Comércio Ltda, Petrópolis/RJ) manipulado de acordo com instruções do fabricante. Após a reação de presa, o molde foi removido para a obtenção do modelo de trabalho em gesso-pedra (Herodent Sodi-Rock, Vigodent S/A Indústria e Comércio, Rio de Janeiro/RJ). 
A mordida construtiva de trabalho foi obtida com o avanço da posição mandibular para anterior, permitindo a realização do mecanismo de ação do Bionator - o que levaria a língua a uma posição mais anteriorizada, juntamente com a mandíbula. Esta obtenção da mordida construtiva foi executada com uma lâmina de cera $n^{\circ} 7$ (Wilson, Polidental Indústria e Comércio de Cotia, Cotia/SP) que era dobrada em três partes no sentido do maior comprimento. Com o auxílio de uma lamparina a álcool, obtinha-se uma forma semelhante à de uma ferradura, sendo levada à boca do paciente para realizar a impressão da oclusão em uma posição mais anteriorizada, porém confortável, seguindo as recomendações de Fantini ${ }^{6}$ : avançar a mandíbula em $5 \mathrm{~mm}$, inicialmente, podendo-se chegar até $75 \%$ da protusão máxima.

O conjunto de modelos de trabalho em gesso superior e inferior, ocluídos através da mordida em cera construtiva, foi enviado a um laboratório de prótese para montagem em articulador Bio Art (São Carlos/SP) e confecção do aparelho Bionator de Balters (Fig. 2) com resina acrílica termopolimerizável e fios de aço.

\section{Instalação e utilização do dispositivo}

O aparelho Bionator de Balters foi instalado e ajustado na boca do paciente. Recomendou-se a

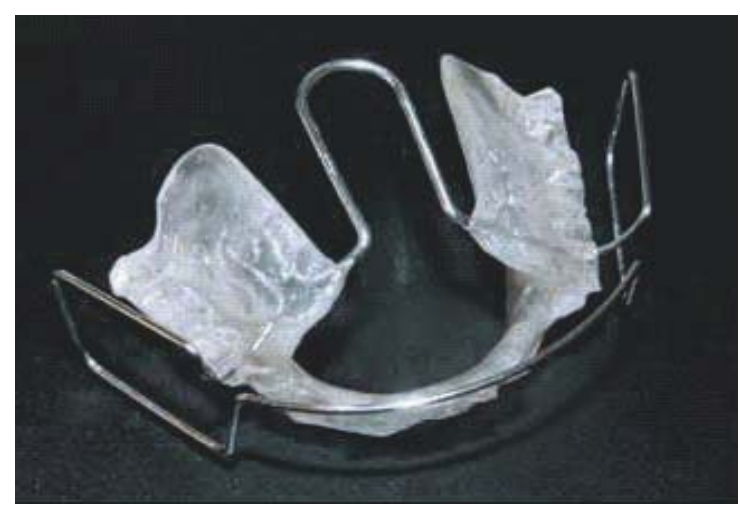

FIGURA 2 - Aparelho Bionator de Balters. utilização do aparelho somente durante o sono, à noite, devendo-se removê-lo pela manhã ao acordar, por um total de 30 dias.

Decorridos os 30 dias, o paciente retornava ao consultório para avaliação do uso do aparelho. Caso a utilização fosse positiva, o paciente era encaminhado para realizar uma nova polissonografia.

\section{ANÁLISE ESTATÍSTICA}

Os dados foram organizados e avaliados por meio de estatística descritiva e tabelas de distribuição de freqüências. A comparação entre $o$ resultado da polissonografia para o IDR inicial e final foi realizada utilizando-se o teste $t$ pareado $(\alpha=0,05)$.

\section{RESULTADOS}

Dos 16 pacientes que iniciaram o tratamento, apenas 9 ( 3 do gênero feminino e 6 do masculino) terminaram o tratamento, por conseguirem utilizar o aparelho.

No gráfico 1, pode-se observar a média e desvio-padrão para a variável IDR (polissonografia). $\mathrm{Na}$ amostra inicial, a média foi de 15,5 (desviopadrão $=15,6$ ). Para a amostra inicial (parcela de pacientes que terminou o tratamento) a média inicial foi de 11,5 (desvio-padrão $=5,0$ ), sendo o valor médio desta variável ao final do tratamento

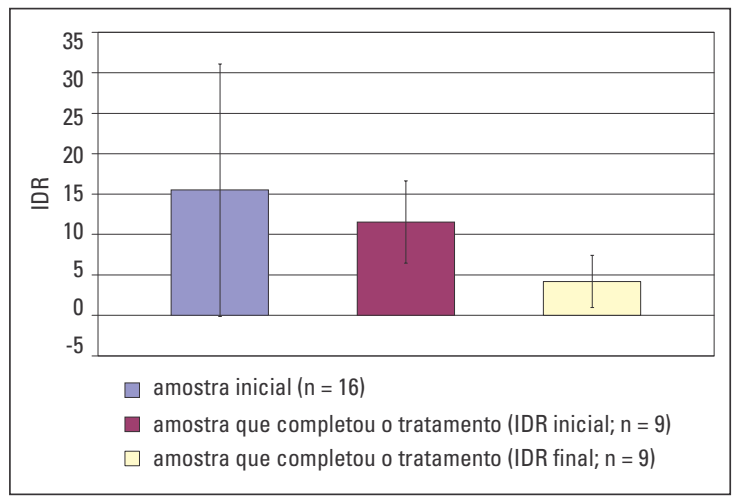

GRÁFICO 1 - Média e desvio-padrão para a variável IDR (polissonografia), segundo a fase do tratamento. 
de 4,2 (desvio-padrão = 3,2). Através do teste $t$ pareado, pode-se observar uma diminuição estatisticamente significativa nos valores de IDR com o tratamento $(\mathrm{p}<0,05)$.

\section{DISCUSSÃO}

O conhecimento do tratamento da Síndrome da Apnéia Obstrutiva do Sono (SAOS) através dos dispositivos intrabucais tem se mostrado importante, devido à multidisciplinaridade da doença, bem como à eficácia que os dispositivos têm apresentado, necessita-se de um posicionamento correto do cirurgião-dentista frente a este problema ${ }^{8}$.

Durante o sono, quando o tônus dos tecidos da orofaringe diminui, estes se aproximam, bloqueando a passagem de $\operatorname{ar}^{8}$. A obesidade, as retrusões mandibulares e as micrognatias, geralmente, determinam ou agravam o caso. Sendo assim, recomenda que seja realizado abrangente exame físico em busca de fatores anatômicos que possam levar ao ronco ou à SAOS ${ }^{32}$. Devem, ainda, ser realizados modelos de estudo e exame facial, para se avaliar o tamanho do pescoço e a possível retrusão mandibular. O IMC do paciente deve ser investigado, havendo forte correlação entre obesidade e a SAOS. Desta maneira, foram selecionados para a pesquisa aqueles que apresentavam IMC entre 20 e 29.

Outro critério de inclusão considerado na seleção dos pacientes para a pesquisa foi a realização de uma consulta prévia com um médico otorrinolaringologista. Esse fator foi importante para se diagnosticar a presença da SAOS, bem como verificar a ausência de problemas nas vias aéreas superiores e visualização de língua em posição posteriorizada, através da endoscopia nasofaríngea rígida e flexível - com realização de manobra de Muller em posição sentada e supina ${ }^{5}$. Há indivíduos que apresentam associação entre ronco noturno e um outro importante problema respiratório: a síndrome da resistência das VAS (vias aéreas superiores) ${ }^{14}$. Nestes, embora não ocorram episódios apnéicos reais, o ronco é tão alto e a respiração é tão trabalhosa que resultam em numerosos despertares durante a noite, deixando os portadores cansados durante o dia, podendo ser confundida com a SAOS.

Dos 16 indivíduos diagnosticados com a SAOS, verificou-se a presença de, pelo menos, 10 dentes em cada arco dentário e seu correto posicionamento, uma vez que se contra-indicam o uso de aparelhos intrabucais em pacientes com quantidade insuficiente de elementos dentários e em posição inadequada nos arcos para ancoragem e retenção da base do aparelho ${ }^{8,10}$, bem como ausência de sintomatologia dolorosa ou ruidosa na $\mathrm{ATM}^{8,30}$.

Apesar dos traçados cefalométricos oferecerem indicadores da possibilidade de sucesso do tratamento da SAOS com o uso de dispositivos intrabucais (por meio dos quais poderiam ser avaliados o tamanho do palato mole, a inclinação do plano mandibular, a posição do hióide, o tipo e a altura facial, e a relação de bases ósseas), estes apresentam limitações (por fornecerem informações apenas em duas dimensões ${ }^{28}$ ), além do que a avaliação cefalométrica não possibilita confirmar o diagnóstico da SAOS. Também se observa um maior aumento do volume dentro das vias aéreas com a utilização do aparelho no sentido laterolateral do que no ântero-posterior, bem como uma modificação das estruturas (devida ao tônus muscular durante o sono), não-verificáveis com a utilização de cefalometria ${ }^{8}$.

Com a realização da polissonografia, determina-se a severidade da apnéia através do índice de apnéia/hipopnéia (IAH) que é o fator principal na seleção e decisão do tratamento. Esta também possibilita o diagnóstico diferencial entre apnéia obstrutiva e central, uma vez que os aparelhos são mais bem indicados nos casos de apnéia obstrutiva leve e moderada ${ }^{33}$, com IAH até 30 , em pacientes retrognatas, não muito acima do peso, e como coadjuvante em outros tratamentos ou quando estes não mostram bons resultados ${ }^{8}$.

Para a determinação do tipo de aparelho a ser 
adotado neste experimento, levou-se em consideração o estudo de Sergi e Zentner ${ }^{27}$, onde foi demonstrado que o Bionator foi o dispositivo melhor aceito pelos pacientes. Além disso, suas características se adequam ao mecanismo de ação que os aparelhos intrabucais devem apresentar para o tratamento da SAOS. Freitas et al. ${ }^{7}$ citaram como vantagens a facilidade de utilização, por ser menor e mais elástico, bem como um posicionamento anteriorizado da mandíbula com os incisivos em topo-a-topo. Este aparelho propicia o fechamento de lábio, trazendo o dorso da língua em contato com o palato mole; aumenta o espaço bucal através da anteriorização da mandíbula, tornando possivel uma melhor posição da língua e proporcionando um melhor relacionamento entre os maxilares, língua, dentes e tecidos moles circundantes; bem como um aumento da dimensão vertical de oclusão, sendo um dispositivo indicado para o tratamento da $\mathrm{SAOS}^{28}$.

Dos 16 pacientes que utilizaram o aparelho ortopédico Bionator de Balters, verificou-se que apenas nove apresentaram boa aceitação $(56,25 \%)$. Os outros 7 pacientes não deram continuidade ao tratamento, pois alegaram desconforto durante a utilização. $O$ índice de aceitação pode ser considerado baixo, quando comparado ao do estudo clínico de Battagel et al. ${ }^{3}$, que verificou um índice de cooperação de $76 \%$. O desconforto alegado pelos pacientes ao utilizarem o aparelho refere-se a dificuldades de adaptação devidas aos efeitos colaterais produzidos pelo uso do Bionator de Balters. Como exemplo, a pressão do fluído sinovial após a protrusão mandibular aumenta, mas retorna após duas horas ao nível norma ${ }^{34}$. Além disto, observa-se salivação excessiva no início do tratamento, devido à nova postura lingual e à presença do aparelho na boca; pequeno desconforto ou cansaço da musculatura mastigatória pela manhã e uma pressão uniforme sobre os dentes, principalmente sobre os anteriores, sobre a gengiva, língua ou maxilares. Pequenos desajustes oclusais ou sensação de não conseguir morder pela manhã são comuns, não devendo perdurar por mais de 30 minutos após a remoção do aparelho ${ }^{30}$. Além disto, observou-se uma expectativa de resultado quase imediata, quando se refere à diminuição do ronco, apesar de se verificar que esta diminuição é variável entre os indivíduos. Pode-se notar, em alguns casos, a diminuição imediata do ronco com a instalação do aparelho e, em outros casos, somente após um determinado tempo de uso. Além disso, sugere-se o uso sistemático deste dispositivo todas as noites, o que poderia conformar os tecidos da região flácida e corrigir seu posicionamento, eliminando a possibilidade de vibração, mesmo sem o uso do aparelho. Em alguns casos, isto pode vir a acontecer, o que seria um motivo a mais para se continuar estudando esta alternativa de tratamento ${ }^{25}$.

Com a utilização habitual do dispositivo, os pacientes que chegaram ao final do tratamento foram encaminhados para uma nova polissonografia, a qual foi realizada com o paciente fazendo o uso do aparelho ortopédico Bionator de Balters para avaliar e quantificar a eficácia deste dispositivo no tratamento do ronco e da apnéia. Observou-se melhora estatisticamente significante em relação ao IDR (índice de distúrbio respiratório), mostrando a efetividade deste dispositivo e corroborando outros estudos ${ }^{1,9,12,13,15,17,26,33}$ que também utilizaram dispositivos intrabucais para o tratamento da SAOS. Este exame, assim como no inicial, realiza uma monitorização das características do sono em condições o mais próximo possivel das normais e semelhantes às do paciente em sua casa. Para isso, o ambiente deve ser agradável, com cama, temperatura, luminosidade e atenuação de sons adequadas, em ambiente que o paciente se sinta relaxado, com bom relacionamento com a equipe de monitorização, incluindo técnicos, enfermagem e médico ${ }^{16}$.

Assim, a utilização do Bionator de Balters pode ser considerada um método de tratamento da SAOS em casos leves ou moderados, após diagnóstico médico, ou ainda ser uma alternativa 
ou complemento aos procedimentos cirúrgicos. Entretanto, verifica-se a necessidade de pesquisas quanto à estrutura e forma mais adequada para que o dispositivo seja mais confortável e bem tolerado pelo paciente.

\section{CONCLUSÃO}

Conclui-se que o aparelho intrabucal Bionator de Balters mostrou-se bastante eficiente para estes pacientes, no que se refere ao tratamento para diminuição do ronco e da apnéia, pois foi observada uma diminuição estatisticamente significante nos valores de IDR com o tratamento.

\title{
The effectiveness of the Balters Bionator orthopedic appliance for the treatment of the Obstructive Sleep Apnea Syndrome
}

\begin{abstract}
Aim: to assess the effectiveness of the Balters Bionator orthopedic appliance for the treatment of the Obstructive Sleep Apnea Syndrome (OSAS). Methods: Sixteen patients (18.8\% women and $81.3 \%$ men) that presented OSAS on diagnosis were selected. Of the 16 patients that used the Balters Bionator orthopedic appliance, only 9 presented good acceptance (56.25\%) and concluded the treatment. They were submitted to another polysomnography exam, performed with the patients using the Balters Bionator orthopedic appliance, in order to assess the effectiveness of this device in treating apnea snoring. Comparison between the results of the initial (11.5) and final (4.2) RDI (respiratory disturbance index) was made by the paired t test $(\alpha=0.05)$. Results: A statistically significant improvement in the RDI was observed after the device was used. Conclusions: The use of the Balters Bionator orthopedic appliance may be considered as a method for treating OSAS in light or moderate cases, in spite of being a method hardly tolerated by patients.
\end{abstract}

Key words: Balters Bionator. Apnea. Snoring.

\section{REFERÊNCIAS}

1. BUÑO AROSTEGUI, A. G.; LORENZO, D. et al. Tratamiento del ronquido y de las apneas del sueno obstructivas com dispositivo de ortosis dental. An. Fac. Odontol., Montevideo, v. 28 , p. $27-41$, abr. 1997.

2. BARBOSA, R. C. Tratamento da síndrome da apnéia obstrutiva do sono e ronco por meio de aparelho reposicionador mandíbulo-lingual: avaliação dos resultados por exames polissonográficos e de imagens obtidas por ressonância magnética. 1999. Tese (Doutorado)-Faculdade de Odontologia, Universidade de São Paulo, São Paulo, 1999.

3. BATTAGEL, J. M.; JOHAL, A.; L'ESTRANGE, P. R. et al.

Changes in airway and hyoid position in response to mandibular protrusion in subjects with obstructive sleep apnoea (OSA). Eur. J. Orthod., Oxford, v. 21, no. 4, p. 363376, Aug. 1999

4. CARDOSO, R. J. A. GONCALVES, E A. N. Tratamento da síndrome da apnéia do sono obstrutiva e ronco noturno e sua influência no tratamento ortodôntico. In: CARDOSO, R. J. A.; GONÇALVES, E. A. N. Odontologia, arte, ciência e técnica: Ortodontia e Ortopedia Funcional. São Paulo: Artes Médicas, 2002. v. 7. p. $185-216$.
5. COLOMBINI, N. E. P.; PINTO, J. A. Síndrome da apnéia do sono obstrutiva: um novo desafio médico-odontológico. JBO: J. Bras. Odontol. Ortop. Maxilar, Curitiba, v. 1, n. 4, p. 57-60, jul./ago. 1996.

6. FANTINI, S. M. Tratamento da síndrome da apnéia do sono obstrutiva e ronco noturno e sua influência no tratamento ortodôntico. In: CARDOSO, R. J. A.; GONÇALVES, E. A. N. Ortodontia e Ortopedia Funcional. São Paulo: Artes Médicas, 2002. v. 7. p. 185-199.

7. FREITAS, M. R.; CONTI, A. C. C. F.; CONTI, P. C. R. et al. Bionator e as desordens temporamandibulares (DMT): mito ou realidade? Rev. Dental Press Ortodon. Ortop. Facial Maringá, v. 5, n. 5, p. 80-84, set./out. 2000.

8. GODOLFIN, L. R. O tratamento do ronco e apnéia do sono com dispositivos intra-orais. Ortodontia, São Paulo, v. 35, n. 2, p. 87-91, abr./jun. 2002.

9. HANS, M. G.; NELSON, S.; LUKS, V. G. et al. Comparison of two dental devices for treatment of obstructive sleep apnea syndrome (OSAS). Am. J. Orthod. Dentofacial Orthop., St. Louis, v. 111, no. 5, p. 562-570, May 1997. 
10. ITO, R. T.; MELO, A. C. M.; BRONZI, E. S. et al. Aparelho anti-ronco: um enfoque multidisciplinar. Rev. Dental Press Ortodon. Ortop. Facial, Maringá, v. 5, no. 2, p. 48-53, mar./ abr. 2000

11. KOHLER, J. F. W.; BRADASCH, V. L. O papel do ortodontista na apnéia obstrutiva noturna. Dens, Curitiba, v. 10, p. 19-28, 1994

12. LAMONT, J.; BALDWIN, D. R.; HAY, K. D et al. Effect of two types of mandibular advancement splints on snoring and obstructive sleep apnoea. Eur. J. Orthod., Oxford, v. 20, no. 3, p. 293-297, June 1998.

13. LAMONT, J.; BALDWIN, D. R.; HAY, K. D. et al. O efeito de dois tipos de splints para avanço mandibular sobre o ronco e a apnéia obstrutiva do sono. Rev. Dental Press Ortodon. Ortop. Facial, Maringá, v. 4, n. 2, p. 74-75, mar./abr. 1999.

14. LEVY, P.; PEPIN, J. L.; MAYER, P. et al. Management of simple snoring, upper airway resistance syndrome, and moderate sleep apnea syndrome. Sleep, Westchester, IL, v. 19, n. 9, p. S101-110, Nov. 1996. Suppl.

15. LIU, Y.; ZENG, X.; FU, M. et al. Effects of a mandibular repositioner on obstructive sleep apnea. Am. J. Orthod. Dentofacial Orthop., St. Louis, v. 118, no. 3, p. 248-256, Sept. 2000

16. LOPES FILHO, O. Polissonografia. In: REIMÃO, R. Tratado de Otorrinolaringologia. São Paulo: Rocca; 1994. p. 215-219.

17. LORIATO, E. A. Dispositivo anti-ronco. Rev. Bras. Odontol., Rio de Janeiro, v. 56, n. 2, p. 73-74,1999.

18. MAGRO FILHO, O.; RANGEL-GRARCIA JÚNIOR, I.; MAGROERNICA, N. et al. Cirurgia ortognática para tratamento da síndrome da apnéia obstrutiva do sono: relato de caso clínico. Rev. Dental Press Ortodon. Ortop. Facial, Maringá, v. 6, n. 5 p. 91-96, set./out. 2001.

19. $M A N C I N I, M . C .: A L O ́ E, F$ Obesidade, apnéia obstrutiva do sono e distúrbios respiratórios. In: HALPERN, A.; MATOS, A. F. G.; SUPLICY, H. L.; MANCINI, M. C.; ZANELLA, M. T. (Org.) Obesidade. 1 ed. São Paulo: Lemos Editorial, 1998. v. 1 , p. 153-170.

20. MANGABEIRA-ALBERNAZ, P. L. M. Síndrome da apnéia obstrutiva do sono e do ronco. In: MANGABEIRA-ALBERNAZ, P. L.; GANANÇA, M. M.; FUKUDA, Y.; MUNHOZ, M. S. L. Otorrinolaringologia para o clínico geral. São Paulo: Fundo Editorial Byk, 1997. p. 169-170.

21. MARCONDES, M.; SUSTOVICH, D. R.; RAMOS, O. L. Tórax e aparelho respiratório. 3. ed. Rio de Janeiro: Guanabara Koogan, 1976. p. 39.
22. MORRISON D L: LAUNOIS, S. H. ISONO, S. et al. Pharyngeal narrowing and closing pressures in patients with obstructive sleep apnea. Am. Rev. Respir. Dis., New York, v. 148, no. 3, p. 606-611, Sept. 1993.

23. PAULIN, R. F.; MELO, A. C. M.; ITO, R. T. et al. A apnéia obstrutiva do sono: considerações gerais e estratégias de tratamento. JBO: J. Bras. Ortodon. Ortop. Facial, Curitiba, v. 6, n. 36, p. $448-492,2001$

24. RAMOS, L. V. T.; FURQUIM, L. Z. Aparelho para apnéia obstrutiva do sono. Rev. Clin. Ortodon. Dental Press, Maringá, v. 3, n. 2, p. 21-26, 2001.

25. RIBEIRO, A. I. $\mathbf{1 0 0}$ motivos para ir ao dentista: o livro do paciente. In: . Para tratar do ronco e apnéia. Curitiba: Maio, 2001. p. 50-51.

26. ROSE, E.; STAATS, R.; VIRCHOW, C. et al. A comparative study of two mandibular advancement appliances for the treatment of obstructive sleep apnoea. Eur. J. Orthod., Oxford, v. 24 no. 2, p. 191-198, Apr. 2002

27. SERGI, H. G.; ZENTNER, A. A comparative assessment of acceptance of different types of functional appliances. Eur. J. Orthod., Oxford, v. 20, no. 5, p. 517-524, Oct. 1998

28. SGARBI, S. R. S.; SGARBI, J. A. Síndrome da apnéia obstrutiva do sono: uma nova visão. JBO: J. Bras. Ortodon. Ortop. Facial, Curitiba, v. 3, n. 16, p. 71-79, 1998.

29. SHEPARD JR., J. W. ; THAWLEY, S. E. Localization of upper airway collapse during sleep in patients with obstructive sleep apnea. Am. Rev. Respir. Dis., New York, v. 141, no. 5, p. 1350-1355, May 1990

30. SILVA, A. B. Os distúrbios do sono podem ser diagnosticados precocemente? In: CARDOSO, R. J. A. Ortodontia e Ortopedia Funcional. São Paulo: Artes Médicas, 2002. p. 201203.

31. SILVEIRA, M. A. Síndrome da apnéia obstrutiva do sono, o ronco e seu tratamento com o aparelho Apnout. JBO: J. Bras. Ortodon. Ortop. Facial, Curitiba, v. 6, n. 32, p. 151-154, 2001

32. VEIS, R. W. Snoring and obstructive sleep apnea from a dental perspective. J. Calif. Dent. Assoc., Califórnia, v. 26, no. 8 , p. 557-565, Aug. 1998

33. WADY, M. H. A.; VARGAS NETO, J.; VEDOVELLO FILHO, M. et al. Placas oclusais no tratamento da síndrome da apnéia obstrutiva do sono: uma alternativa conservadora. Ortodontia, São Paulo, v. 35, n. 2, p. 137-144, abr./jun. 2002.

34. WARD, D. M.; BEHRENTS, R. G.; GOLDBERG, J. S

Temporomandibular synovial fluid pressure response to altered mandibular positions. Am. J. Orthod. Dentofacial Orthop., St. Louis, v. 98, no. 1, p. 22-28, July 1990.
Endereço para correspondência

Paulo Alberto Donadelli Nabarro

Rua Santos Dumont, 3.472 - sala 101

CEP: 87.013-050 - Maringá/PR

E-mail: paulonabarroorto@zipmail.com.br 